Vol. 1, No. 1, June 2021, Pp. 4-11

ISSN: 2693-9797 (online)

\title{
Consistency in Healthcare Services and Outcomes for Refugees in the United States: Barriers and Potential Solutions
}

\author{
Muzzammil Ahmadzada, Johns Hopkins University \\ Waqas Haque, University of Texas Southwestern Medical Center \\ Ahmad Ahmadzada, School of Optometry, University of California, Berkeley
}

\begin{abstract}
A large percentage of the nearly 3.1 million newly arrived refugees in the United States (U.S.) face difficulties in securing better overall health outcomes for themselves and their families, to the extent that many experience an exacerbation of pre-existing conditions due to post-migration stressors. Despite presenting with more chronic health conditions and lower overall health than the average American patient, certain barriers exist that prevent refugees from having consistent treatment and access to healthcare services. These barriers include limited screening upon entry, short duration of resettlement assistance, and linguistic and cultural differences with their healthcare providers. To combat these barriers and improve health outcomes for refugee patients, we propose and discuss the expansion of screening upon entry into the U.S., the extension of the assigned refugee assistance period, and the creation of a federal certification program that works to produce cultural liaisons that can help with medical interpretation and cultural mediation.
\end{abstract}

\section{Introduction}

As per Article 1 of the 1951 Refugee Convention in Geneva, Switzerland, a refugee is: a person who is outside his/her country of nationality, has a well-founded fear of persecution because of his/her race, religion, nationality, membership in a particular social group or political opinion, and is unable or unwilling to return due to fear of persecution (UNHCR 2001, 1). There are over 80 million people worldwide who meet these criteria in 2020, with 43\% (34 million) of refugees being below the age of 18 (UNHCR 2020, 1). The 1951 Refugee convention was the first international agreement to outline the basic rights of refugees to be maintained in host countries, including access to the same quality of health as the host populations (UNHCR, 1). Healthcare access is especially difficult for refugees owing to the mental and physical trauma in their countries of origin. This demographic has been the result of discrimination in many countries especially during the coronavirus pandemic, which has also worsened job prospects and the ability to receive medical treatment (Barbiroglio 2021, 1).

The United States is an example of a host country that welcomes many of these displaced individuals, irrespective of their area of origin or background, to start a new life within its borders. Since the Refugee Act was passed in 1980, more than 3.1 million refugees have been admitted into the United States by the year 2021 (U.S. Dept. of State, 1). Unfortunately, a large percentage of these refugees experience challenges in securing better overall health 
outcomes for themselves and their families, to the extent that many experience an exacerbation of pre-existing conditions due to post-migration stressors. In this essay, we investigate issues related to the arrival and resettlement processes in the United States that may benefit from targeted interventions to mitigate both short and long-term health concerns in chronically ill refugee populations.

\section{Refugees and Chronic Illness}

Many of the leading refugee countries of origin have observed a dramatic increase in the rates of chronic non-communicable diseases (NCDs) among their residents, possibly owing to a combination of factors including an aging population and transitioning to sedentary urban living (KFF 2019). Reports from the World Health Organization in 2021 show that chronic NCDs are responsible for 41 million deaths every year-equivalent to $71 \%$ of all deaths globally - with $77 \%$ of all NCD deaths being in low and middle-income countries (WHO 2021). A retrospective study showed that $60 \%$ of adult refugees had at least one chronic NCD and $37 \%$ had two or more NCDs (Taylor 2014, 4). NCDs that refugees were commonly found to be diagnosed with are included in Table 1.

\begin{tabular}{|c|c|}
\hline \multicolumn{2}{|c|}{$\begin{array}{c}\text { Chronic non-communicable diseases } \\
\text { found in adult refugee populations }\end{array}$} \\
\hline Type 2 Diabetes & $51.8 \%$ \\
\hline Hypertension & $72.2 \%$ \\
\hline Dyslipidemia & $27.5 \%$ \\
\hline Cerebrovascular disease & $26.1 \%$ \\
\hline
\end{tabular}

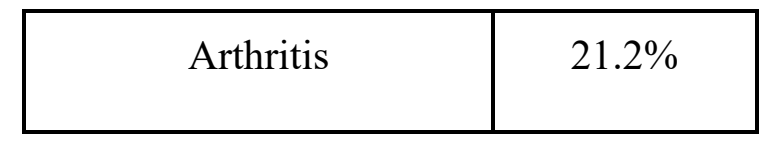

Table 1 (Boulle et. al 2019, 1; Pour 2014, 1; Doocy et. al 2016, 5)

In addition to their physical health, refugee populations tend to experience severe behavioral health issues as well, with an average of $30.6 \%$ being diagnosed with post-traumatic stress disorder (PTSD, prevalence ranging from $3 \%$ to $86 \%$ ) and an average of $30.8 \%$ being diagnosed with major depression (prevalence ranging from $3 \%$ to $80 \%$ ) (Terasaki et. al 2015,7 ). This has led to cases such as the group of newly arrived Bhutanese refugees between 2009 and 2012 for whom the estimated annual suicide rate was 24.4 per 100,000 persons, nearly double that of the overall United States population (Terasaki et. al 2015, 7). Despite lower health on average as a group, numerous refugees to this day experience challenges when trying to secure consistent treatment for their chronic NCDs. This is exacerbated further by a lack of health literacy in refugee communities, as shown in a 2018 study of refugees and asylum seekers (Chuah et al 2018, 6).

\section{Limited Screening Upon Entry}

For a refugee to be admitted into the U.S., they must first pass an initial health screening to ensure that they do not carry certain infectious diseases, including tuberculosis, malaria, and HIV (Barnett 2004, 1). This medical screening is performed first overseas, and then upon arrival to the United States by a civil surgeon-a physician employed by the United States Citizenship and Immigration Services (Downes 2011, 1). However, this screening done by the civil surgeon only screens for a very limited number of chronic conditions, since they are not imminent public health threats, and is optional (CDC 
2021). Despite a high proportion of refugees electing to be screened within their first 30 days, the fact that the screening is optional allows for many refugees to remain unscreened. Thus, some infectious illnesses, previous traumatic injuries, and other chronic NCDs often go undetected in this refugee population (Downes 2011, 1).

A telling example of under-screening is the case of a 55-year-old refugee from Burundi who came to the U.S. with his family. After experiencing consistent trouble completing basic tasks and thus continuously being fired from his jobs, one of his employers finally decided to send him for an occupational examination to determine if there was anything wrong with his health. His overall findings were unremarkable except for his visual acuity, which tested at 20/25 in his right eye and 20/200 in his left. He was later diagnosed with onchocerciasis (Downes 2011, 1), a relatively uncommon cause of permanent blindness that occurs in 20.9 million people worldwide (WHO 2019, 1). This is one of many examples of refugees whose chronic conditions go unnoticed, undiagnosed, and thus untreated because they were not offered any general examinations to determine the overall status of their health. By improving access to preventative healthcare, downstream consequences on the native country's healthcare system can be lessened, such as emergency hospitalizations as refugees become symptomatic or are reinfected with diseases that have been otherwise steadily decreasing in the native population, like tuberculosis or gastrointestinal parasites (Barnett 2004, 3).

\section{Short Duration of Assigned Resettlement Assistance}

Once a refugee has passed the health screening and is allowed entry into the U.S., the State Department assigns them to one of nine refugee resettlement agencies (RRAs), all funded by the U.S. Department of State's Bureau of Population. The RRA is then responsible for assisting the refugee in acquiring basic living necessities — such as housing and food arrangements, clothing, employment counseling, medical care, and enrolling children in school-for the first 90 days of their time in the U.S. After the 90 days are over, the RRA's caseworkers move on to serving other individuals and the refugee is left to make his or her own decisions (Gilmer 2018, 34). This has been a significant problem for many refugee families who experience difficulties in assimilating to living in the U.S. within a brief three months. Many may have not even learned basic English during that time span. An RRA manager in Los Angeles, one of the most refugee-dense cities in the nation, said:

"We know that many health conditions [are not detected] within those 90 days. But even those that [are], we're not going to respond after 90 days. So if we have a refugee who has a health condition who is dragging her feet in getting care, past the 90 day point? We know she still needs it; how are we going to make sure she gets it?...Y You have to remember we have a 90-day window to work with refugees. So what happens is refugees will stop seeking medical care." (Gilmer 2018, 35).

After a refugee loses their caseworker, many are left overwhelmed and anxious with the thought of having to take on all the tasks the caseworker was helping them with, such as scheduling doctor appointments, navigating their insurance plans, and learning about the medication they need to take. It can be due to not owning a car or being unfamiliar with the public transportation system in the area, thus barring them from even making it to their appointments if they did schedule one. It can also be a result of not knowing how 
and when to fill out the proper paperwork to continue seeing a doctor. Without a dedicated caseworker on board, seemingly minute tasks can become insurmountable barriers for addressing their health conditions.

\section{Language and Cultural Differences}

As briefly mentioned earlier, another major issue refugees face is the language and cultural barrier between them and their care providers (Schimmel 2020, 1). Acculturation challenges and lack of access to culturally appropriate care has been the most frequently cited barrier to care in each region and for most refugee groups (Gilmer $2018,24)$. In terms of language, refugee patients who did not speak English were either unable to fully convey their health situation and/or fully understand the response, or they were unable to develop trust for their providers due to feelings of isolation and frustration. In terms of culture, healthcare providers who had no insight as to the culture of their refugee patients often missed out on opportunities to be supportive of their patients in ways that made them feel heard, understood, and comfortable. Instead, the lack of cultural understanding led to mistrust between the patient and provider and medication noncompliance.

The language barrier is increasingly seen to have dangerous effects on refugee populations. An example of this is the case of a refugee who assumed that his mother's heart medication was Aspirin, the only medicine he was familiar with from his home country, and gave it to her every day for headaches. Another example is the case of a provider in Tucson, Arizona who assumed that the patients understood how a bottle of lotion was to be used. Instead, there were two separate cases of refugee patients that ended up being hospitalized for drinking multiple bottles of lotion (Gilmer 2018, 25).
Culturally humble and competent care is also being pushed to the forefront of health concerns for refugee populations, as it too can result in dangerous health situations. A hospital physician in Seattle spoke on the issue, stating:

"If you have a lot of Muslim patients and Ramadan is coming up and there is a lot of diabetes in those patients, figuring out how you would talk to your fasting diabetic patients about Ramadan is important," (Gilmer 2018, 25).

A survey of 45 primary care physicians in Canada found that while a majority agree that knowledge of patients' religious obligations is important to optimizing health recommendations, many were unaware that water and medication pills cannot be consumed during the fast of Ramadan that Muslims partake in (Hamodat et al 2020, 2). If physicians and other healthcare providers are not aware of their patients' cultural and religious beliefs when prescribing treatment options, this may foster confusion and potentially mistrust among refugee patients.

Another example is the case of a refugee mother in Tucson who refused to call an ambulance during a medical emergency despite seeking help. When someone finally confronted her and told her to call 9-1-1, she responded "Why would I call 9-1-1 for my child? Why would I call the police for him?" (Gilmer 2018, 25). This example demonstrates how the difference in culture for refugees often leads to misunderstandings regarding U.S. resources and services. In recent survey studies, it was found that ethnic minorities report being less likely to take the vaccine for COVID-19, reflecting a systematic gap in care and integration (Lacobucci 2021, 1). 


\section{Expansion of Health Screenings Upon Arrival}

There are three solutions proposed in this paper to help mitigate health concerns in refugees diagnosed with chronic conditions as well as address the issues with the U.S.'s current health screening process. As for the issue of the initial health screening that takes place prior to a refugee's entry into the U.S., there is a need for the screening to be expanded to account for more chronic NCDs, any underlying mental and behavioral health issues, and severe trauma. The earlier these conditions are detected, the earlier the refugee can attain consultation and treatment from their healthcare provider, preferably through the assistance of the caseworker. This is also where the bridge between screening and primary care practices is important. One major goal of RRAs is to link individuals and families to on-going timely and high-quality care, and primary care offices with expertise in cross cultural care can provide that (Griswold 2018, 1). By connecting incoming refugees with primary care providers, they can not only have access to more intricate screening, but also to treatment options and guidance for any conditions that the screenings reveal.

Diagnosing and treating chronic NCDs early is not only beneficial for patients, but also for the U.S. healthcare system. Studies show that disease prevention and early treatment is significantly cheaper than late-stage treatment. Considering the $\$ 1.1$ trillion dollar annual cost of treating chronic diseases in the U.S.- $\$ 3.2$ trillion of lost economic productivity is accounted for as well-it is in every party's best interest for chronic NCDs to be detected and treated as early as possible (Waters 2018, 1).

\section{Extending Duration of RRA Support}

Congressional action mandating the extension of the RRA assignment period from 90 days to 24 months has the potential to greatly ease the burden of adjustment and resettlement in refugee communities. Three months is not enough time for people to learn a new language, become familiar with public transport, and develop trusted relationships with their healthcare providers. A recent survey conducted by a UK-based research consultancy revealed that, on average, it takes 24 months to feel fully at home abroad (Davies 2018). Giving caseworkers two years to develop longlasting and meaningful bonds with refugees will allow for adequate screening and tailored treatment as health issues arise. While this would entail additional funding for such programs, both the short- and longterm cost savings from improved health would likely outweigh the administrative expense.

Further, critics may claim that 24 months of RRA assistance is still impractical and would overburden the nine RRAs in America. However, up until the year 2000, RRA assistance was set at 36 months with no major problems arising from the length of follow-up (Gilmer 2018, 35).

Additionally, the average annual number of refugees in the U.S. from 1980 to 2000 , while RRA assistance was set at 36 months, was 97,954 people, ranging from 61,218 to 207,116. From 2001 to 2020, while RRA assistance was set at 90 days, the average annual number of incoming refugees was 52,864 and ranged from 11,814 to 84,995 (National Immigration Forum 2020, 1). These numbers indicate that if the RRAs were able to handle almost double today's average annual refugee resettlement rate for 36 months, 24 months should be feasible. 
Additionally, it is important to mention that RRAs are funded by a per capita model, where the amount of funding depends on the number of refugees served by each RRA (Gilmer 2018, 36). That being said, recent reductions in the cap on refugee admissions to only 15,000 refugees - the lowest number since the 1980 Refugee Act took effect (Miroff 2020) - by the former Trump administration, and the steady decline since the early 2000s before the Trump administration took office, have resulted in major layoffs for the RRAs. Over 300 positions have been cut from seven of the nine RRAs, which does not take into account positions that saw a reduction in their hours (Gilmer 2018, 37). Therefore, in accordance with the proposed solution above, the RRA assistance period should be extended from 90 days to 24 months.

\section{Federal Certification Program}

As for the issue of language and cultural barriers, a potential solution is the creation of a federal certification program certifying cultural liaisons (CLs) in medical translation and basic cultural competency and humility. No such program currently exists on the federal level (Gilmer 2018, 50). Even though healthcare providers are required to provide "qualified" oral interpreters and written translated documents to limited English proficiency patients by the Title VI of the Civil Rights Act of 1964, the Americans with Disabilities Act, and the Affordable Care Act, there is no national standard that deems interpreters "qualified" in medical interpretation and cultural humility (Lingolet 2021). Some states and national associations offer interpreter certification programs, but since providers are not required to use them, they are often neglected. This is due to the financial burden of translation often falling on health facilities and providers that have the least incentive to incur these costs. As part of the program, federal funding can increase the number of translators for federally qualified health centers and as an add-on service for clinics in refugee-dense communities.

CLs would undergo an oral and written examination for the language and culture they specialize in. Once certified, they would accompany refugees to doctor and pharmacy visits to foster trust and mutual understanding between patient and provider. There is a similar program to this in Malta which has successfully developed training for "cultural mediators" to explain and negotiate the cultural beliefs and behaviors related to health, illness, and medical care (WHO 2018, 5).

Ideally, the program would begin by seeking interpreters that are experts in the languages and cultures most common among refugees, and then gradually expand to include as many as possible. By collaborating and coordinating with primary care offices and community health workers, CLs could maximize the integration of inclusive healthcare to all patients, whether they are refugees or not (Gunderson 2018, 334). The creation of this federal certification program that certifies CLs that healthcare providers are required to employ for their translational needs provides a standardized system for basic medical interpretation and cultural competency and humility for all patient populations. This program can also employ refugees who have successfully integrated into society, as well.

Critics may claim that the program will be too costly. However, a 2017 draft report from the Department of Health and Human Services found that over the past decade, refugees have contributed $\$ 63$ billion more in tax revenue than they cost in terms of providing public benefits. Additionally, on a per capita basis, refugees contributed more than U.S.-born citizens during that time period (Breene 2018, 1). Another study 
conducted in Columbus, Ohio found that refugees supported more than 21,000 jobs and contributed upwards of $\$ 1.6$ billion to the local economy (Breene 2018, 1). And for refugees to continue to contribute to the local and national economy, it is important for their tax money to be reinvested into programs such as the language and cultural competency certification program proposed above, so as to ensure consistent highquality healthcare for them and their families.

\section{Conclusion}

In sum, refugees that are diagnosed with a plethora of NCDs could benefit from certain amendments and changes to the current refugee healthcare process. While the coronavirus pandemic and the increasing debt burden of the American economy may make public services an afterthought, policymakers and administrators should continue to focus on developing programs that can represent a substantial health benefit for many communities. By improving the health screening process for refugees entering the country, extending the RRA period to 24 months, and expanding translation services for refugees, noncommunicable disease in refugee populations can be mitigated.

\section{References}

Barbirolgio, Emanuela. 2021. "Migrants and Refugees' Health in the Wake of Covid-19." Osservatorio Balcani Caucaso Transeuropa. January 25, 2021.

https://www.balcanicaucaso.org/eng/Areas/Balkans/ Migrants-and-refugees-health-in-the-wake-of-Covid19-207844.

Barnett, Elizabeth D. 2004. "Infectious Disease Screening for Refugees Resettled in the United States." Clinical Infectious Diseases 39, no. 6: 83341. https://doi.org/10.1086/423179

Boulle, Philippa et al. 2019. "Cardiovascular Disease among Syrian Refugees: A Descriptive Study of
Patients in Two Médecins sans Frontières Clinics in Northern Lebanon." Conflict and Health 13, no. https://doi.org/10.1186/s13031-019-0217-x.

Breene, Tim. 2018. "Refugees Don't Drain America's Economy. They Revitalize It.” Los Angeles Times, February 12, 2018.

https:/www.latimes.com/opinion/op-ed/la-oe-breenerefugees-economy-20180212-story.html.

Chuah, Fiona Leh Hoon et al. 2018. "The Health Needs and Access Barriers among Refugees and Asylum-Seekers in Malaysia: A Qualitative Study." International Journal for Equity in Health 17, no. 1. https://doi.org/10.1186/s12939-018-0833-x.

Davies, Rhiannon. n.d. "Feeling at Home Abroad How Long Does It Take?” British Expatriate Community. http://britishexpats.com/articles/movingabroad/feeling-home-abroad/.

Doocy, Shannon, et al. 2016. "Prevalence, CareSeeking, and Health Service Utilization for NonCommunicable Diseases among Syrian Refugees and Host Communities in Lebanon." Conflict and Health 10, no. 1. https://doi.org/10.1186/s13031-016-0088-3.

Downes, Elizabeth and Anjalie Graham. 2011. "Health Care for Refugees Resettled in the U.S." Clinician Reviews 21, no. 3: 25-31.

https:/www.mdedge.com/clinicianreviews/article/79 718/health-care-refugees-resettled-us?.

National Immigration Forum. 2019. "Fact Sheet: U.S. Refugee Resettlement.” January 25, 2019.

https://immigrationforum.org/article/fact-sheet-u-srefugee-resettlement/.

Gilmer, Christin. 2018. “Assessing Perceived Barriers to Health Care Access for Resettled Refugees in the Western United States." PhD diss. Harvard University. http://nrs.harvard.edu/urn3:HUL.InstRepos:37945656

Centers for Disease Control and Prevention. 2021. "Guidance for the U.S. Domestic Medical Examination for Newly Arriving Refugees." www.cdc.gov/immigrantrefugeehealth/guidelines/do mestic-guidelines.html.

Gunderson, Jean M. et al. 2018. "Community Health Workers as an Extension of Care Coordination in Primary Care." The Journal of Ambulatory Care Management 41, no. 4: 333-340, https://doi.org/10.1097/JAC.0000000000000255.

Hamodat, Hayam et al. 2020. "Primary Care Physicians' Knowledge, Perceptions, and Comfort 
Level in Managing Patients Fasting in Ramadan.” Journal of Primary Care \& Community Health 11: 15. https://doi.org/10.1177/2150132720933796.

Iacobucci, Gareth. 2021. "Covid-19: NHS Must Tackle Vaccine Lies to Improve Uptake among Ethnic Minorities, Says Stevens." BMJ 372: $n 242$. https://doi.org/10.1136/bmj.n242.

Miroff, Nick. 2020. "Trump Cuts Refugee Cap to Lowest Level Ever, Depicts Them on Campaign Trail as a Threat and Burden." Washington Post, October $1,2020$.

www.washingtonpost.com/immigration/trump-cutsrefugee-cap/2020/10/01/a5113b62-03ed-11eb-88797663b816bfa5_story.html.

Pour, Mehdi Sanati et al. 2014. "Prevalence of Dyslipidaemia and Micronutrient Deficiencies among Newly Arrived Afghan Refugees in Rural Australia: A Cross-Sectional Study." BMC Public Health 14. https://doi.org/10.1186/1471-2458-14-896.

Schimmel, Becca. 2020. "Language Barrier Can Cause Healthcare and Housing Obstacles for International Community." WKU Public Radio, February 16, 2020.

https://www.wkyufm.org/post/language-barrier-cancause-healthcare-and-housing-obstaclesinternational-community\#stream/0.

Taylor, Eboni M., et al. 2014. "Physical and Mental Health Status of Iraqi Refugees Resettled in the United States." Journal of Immigrant and Minority Health 16, no. 6: 1130-1137. https://doi.org/10.1007/s10903-013-9893-6.

Terasaki, Genji, Nicole Chow Ahrenholz, and Mahri Z. Haider. 2015. "Care of Adult Refugees with Chronic Conditions." The Medical Clinics of North America 99, no. 5: 1039-58.

https://doi.org/10.1016/j.mcna.2015.05.006.

Lingolet. 2021. "The Laws Requiring Interpreters in Healthcare in 2021." www.lingolet.com/lawhealthcare-interpreters.

The Henry J. Kaiser Family Foundation. 2019. "The U.S. Government and Global Non-Communicable Disease Efforts." March 13, 2019. www.kff.org/global-health-policy/fact-sheet/the-u-sgovernment-and-global-non-communicable-diseases/.

UNCHR. n.d. "Access to Healthcare." Accessed February 11, 2021. https://www.unhcr.org/enus/access-to-healthcare.html.
UNHCR. 2001. "Frequently Asked Questions about the 1951 Refugee Convention." June 1, 2001. https://www.unhcr.org/enus/news/stories/2001/6/3b4c06578/frequently-askedquestions-1951-refugeeconvention.html\#_Toc519482140.

UNHCR. 2020. "Refugee Statistics.” December 8, 2020. https://www.unhcr.org/refugee-statistics/.

U.S. Department of State. n.d. "Refugee Admissions." https://www.state.gov/refugeeadmissions/.

Waters, Hugh and Marlon Graf. 2019. "Chronic Diseases Are Taxing Our Health Care System and Our Economy." STAT, May 31, 2018. https://www.statnews.com/2018/05/31/chronicdiseases-taxing-health-care-economy/.

World Health Organization. 2021.

"Noncommunicable Diseases." April 13, 2021. www.who.int/news-room/factsheets/detail/noncommunicable-diseases.

World Health Organization. 2018. "Onchocerciasis." November 23, 2018. https://www.who.int/newsroom/fact-sheets/detail/onchocerciasis.

World Health Organization Regional Office for Europe. 2018. Report on the Health of Refugees and Migrants in the WHO European Region: No Public Health without Refugees and Migrant Health. World Health Organization.

https://apps.who.int/iris/handle/10665/311347 